\title{
Qualitative Identification of Roseburia hominis in Faeces Samples Obtained from Patients with Irritable Bowel Syndrome and Healthy Individuals
}

\author{
PECYNA Paulina ${ }^{1 *}$, BABALSKA Zuzanna ${ }^{1}$, SZYMENDERA Klaudia ${ }^{1}$, GABRYEL Marcin ${ }^{2}$, \\ MAŃKOWSKA-WIERZBICKA Dorota ${ }^{2}$, NOWAK-MALCZEWSKA Dorota ${ }^{1}$, TOMCZAK \\ Hanna $^{1,3}$, GÓRNA Monika1, CHMIELEWSKA Małgorzata ${ }^{4}$, JAWORSKA Marcelina Maria1, \\ GRZYMISŁAWSKI Marian², DOBROWOLSKA Agnieszka² and GAJĘCKA Marzena ${ }^{1,5}$ \\ ${ }^{1}$ Chair and Department of Genetics and Pharmaceutical Microbiology, Poznan University of Medical \\ Sciences, Swiecickiego 4, 60-781 Poznan, Poland \\ 2 Department of Gastroenterology, Dietetics and Internal Diseases, Poznan University of Medical Sciences, \\ Przybyszewskiego 49, 60-355 Poznan, Poland \\ ${ }^{3}$ Central Microbiology Laboratory, H. Swiecicki Clinical Hospital at the Poznan University of Medical \\ Sciences, Przybyszewskiego 49, 60-355 Poznan, Poland \\ ${ }^{4}$ Chair and Department of Cell Biology, Poznan University of Medical Sciences, Rokietnicka 5D, 60-806 \\ Poznan, Poland \\ ${ }^{5}$ Institute of Human Genetics, Polish Academy of Sciences, Strzeszynska 32, 60-479 Poznan, Poland \\ PECYNA Paulina - pa.pecyna@gmail.com \\ BABALSKA Zuzanna - z.babalska@gmail.com \\ SZYMENDERA Klaudia - klaudiaszymendera@gmail.com \\ GABRYEL Marcin - marcingabryel1@o2.pl \\ MAŃKOWSKA-WIERZBICKA Dorota - dmankowska.wierzbicka@gmail.com \\ NOWAK-MALCZEWSKA Dorota - dmnowak@ump.edu.pl \\ TOMCZAK Hanna - tomczak.hanna@spsk2.pl \\ GÓRNA Monika - monikagorna25@gmail.com \\ CHMIELEWSKA Małgorzata - mchmielewska@ump.edu.pl \\ JAWORSKA Marcelina Maria - marcelinajaworska@ump.edu.pl \\ GRZYMISŁAWSKI Marian - mariangrzym@ump.edu.pl \\ DOBROWOLSKA Agnieszka - agdob@ump.edu.pl \\ GAJĘCKA Marzena - gamar@man.poznan.pl \\ + Presented at the 1st International Electronic Conference on Microbiology, 2-30 November 2020; Available \\ online: https://ecm2020.sciforum.net/
}

Published: 2 November 2020

\begin{abstract}
Various products coded by genes recognized in microbiota are involved in many biochemical pathways in human body. Bacteria composition in the gastrointestinal tract may be an important aspect of selected diseases' pathogenesis, including irritable bowel syndrome (IBS). Traditional research methods based on classical microbiology, using selective media for bacterial growth, have proven to be ineffective. The use of genetic methods allows the identification of unidentified microbiota, including anaerobes. Roseburia hominis is a flagellated gut anaerobic commensal bacterium, producing short-fatty acids. The knowledge about the microbial components of the intestinal ecosystem is still very limited, including Roseburia hominis. The study aimed to identify Roseburia hominis in the faeces samples obtained from IBS patients and healthy individuals, using PCR techniques. The differences between studied groups were observed, R. hominis may play a role in IBS etiology.
\end{abstract}

Keywords: gut microbiome; gut microbiota; Roseburia hominis; IBS 


\section{Introduction}

All the microorganisms inhabiting the particular human body regions or organs are collectively called a microbiome. The human body encompasses several various microbiomes that include specific populations of microorganisms [1]. Currently, intensive research is underway on the microbiomes and their influence on human health. The knowledge about microbiomes is still very limited, including the intestinal microbiome [2].

Nowadays, the gut microbiome is one of the great interests of researchers because of its potential. Bacteria composition in the gastrointestinal tract may be an essential aspect of the pathogenesis of selected diseases. Gut microbial imbalance (dysbiosis) may lead to various diseases, including irritable bowel syndrome (IBS) [3]. Whereas human microbiomes are abundant in unculturable bacteria, and traditional research methods based on classical microbiology have been proved ineffective, it is necessary to characterize in detail their composition to further evaluate the function of particular microbiota [4]. Roseburia hominis is a flagellated gut anaerobic commensal bacterium, producing short-fatty acids. This property is essential in gut motility, immunity maintenance, and anti-inflammatory properties. There are reports suggesting that Roseburia spp. may play a role in IBS's pathogenesis [5]. The knowledge about the microbial components of the intestinal ecosystem is still very limited, including Roseburia hominis.

\section{Methods}

The study protocol was approved by the Institutional Review Board at Poznan University of Medical Sciences. All individuals provided informed consent after the possible consequences of the study were explained, in accordance with the Declaration of Helsinki. The aim of the study was to identify Roseburia hominis in the faeces samples using PCR techniques. The study was conducted on samples obtained from IBS patients (women, $n=70$, and men, $n=50$ ) and individuals without any intestinal symptoms (women, $\mathrm{n}=28$, and men, $\mathrm{n}=23$ ). After bacterial DNA extraction using the spincolumn method (ZymoBIOMICS DNA Miniprep Kit, Zymo Research, USA), DNA concentrations were measured using DeNovix Spectrophotometer (DeNovix, USA) and stored in $-20 \pm 2{ }^{\circ} \mathrm{C}$ for further analyses.

Qualitative identification of Roseburia hominis, based on the amplification of RHOM_14625 and RHOM_14635 gene fragments was performed. PCR products were purified using an ExoSAP-IT for PCR product Clean-Up (Affymetrix, USA), and the specificity was confirmed by Sanger sequencing (sequence reading was performed at Genomed, Poland).

Then, a statistical analysis of the obtained data using the Chi-square test was conducted.

\section{Results and Discussion}

The human digestive tract, especially its distal segment, is colonized by numerous bacteria that create an intricate community called the gut microbiome. Its presence is crucial for maintaining health by preventing gut colonization by pathogens, producing nutrients, and maintaining the integrity of intestinal mucosa [6]. Nowadays, the human gut microbiota is under research to understand better the vast influence on the human body. Some species likely play an essential role in the gut microbiome, especially in some diseases pathogenesis.

$R$. hominis are relatively newly recognized probiotic bacteria species [7], the most proficient butyrate producers [8], and considered as the most mobile species in the gut microbiome [9]. Those bacteria occur predominantly in the colon. R. hominis has the ability to penetrate the mucus layer and stick to the surface of host intestinal epithelial cells, which promotes probiotic potential of these gut bacteria [10].

Roseburia spp. may play a role in gut diseases. Roseburia spp. was observed to be reduced in the gut in individuals affected by inflammatory bowel diseases (IBD) [11,12]. Machiels et al. indicated that the lack of $R$. hominis in the gut microbiome among patients with colitis ulcerosa (UC) was found [13]. What is more, Chassard et al. hypothesized that IBS symptoms were correlated with Roseburia spp. dysbiosis [14]. 
In our study, the assessed RHOM_14625 gene fragments of $R$. hominis were recognized in samples derived from $9(13 \%)$ female and $21(42 \%)$ male IBS patients, and in $15(54 \%)$ and $7(30 \%)$ female and male control individuals, respectively. The difference in the presence of the evaluated gene between healthy individuals and IBS patients was statistically significant, and the p-value was 0.0001 (Fig.1). Considering the presence of the second evaluated gene fragment of RHOM_14635 in R. hominis fragment, the PCR-amplified fragment was detected in $35(50 \%)$ and $33(66 \%)$ samples obtained from female and male patients, respectively, and in $18(64 \%)$ and 7 (30\%) samples from female and male control individuals. The difference in the analyzed gene distribution was statistically significant ( $p$-value $=0.02$ ) (Fig. 2). Previously, Rigsbee et al. reported that Roseburia spp. abundance was the same among healthy children as well as in diarrhea-predominant IBS children in stool samples [15]. However, Chassard et al. reported in detail that Roseburia spp. was reduced among patients with the IBS - constipation subtype comparing to healthy individuals [12]. What is interesting, after IBS treatment, Roseburia spp. abundance in the gut microbiome was found to be comparable to healthy individuals [16,17]. These and other scientific reports suggest considerable complexity of bacterial composition and function in various diseases, including IBS. 

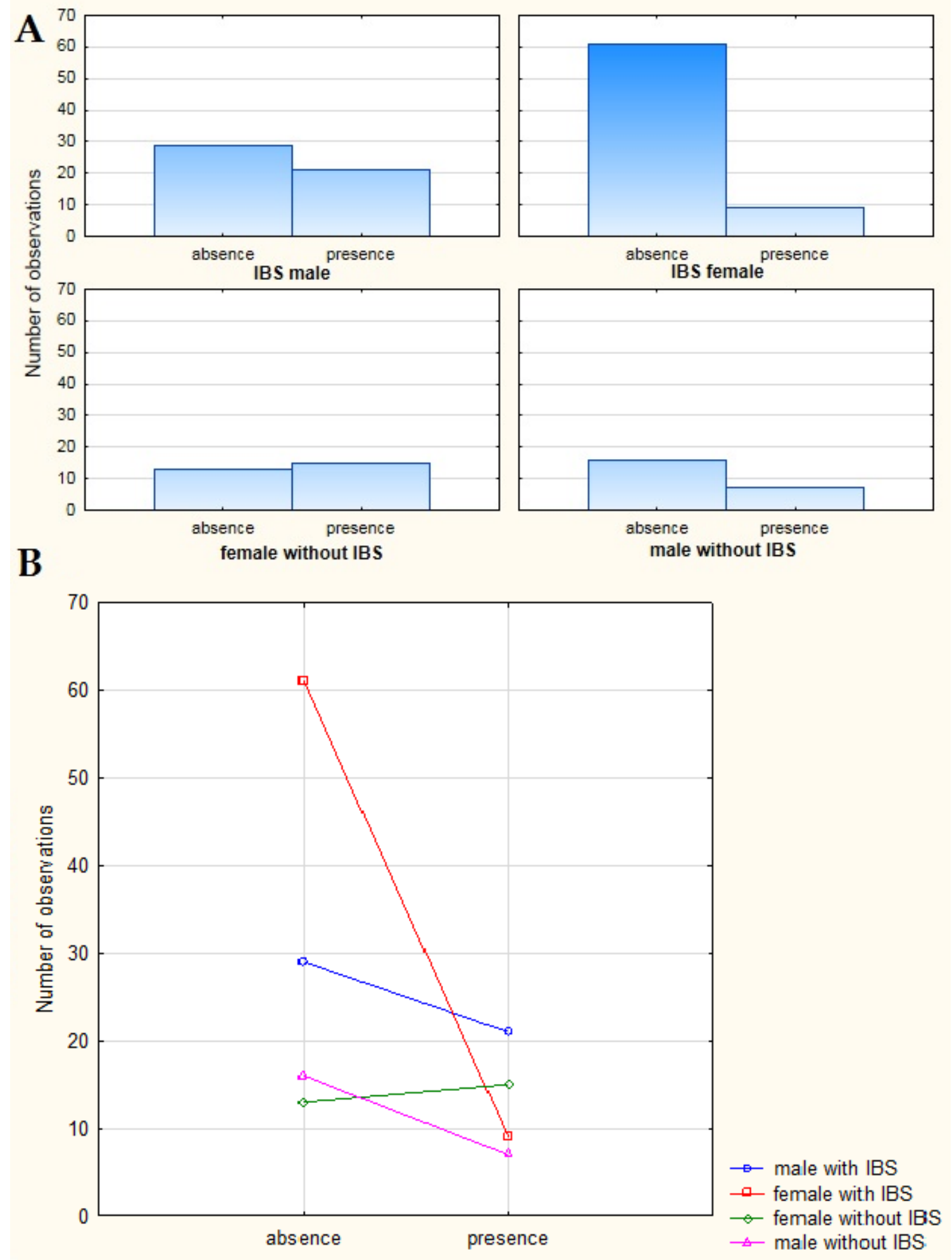

Figure 1. (A) Histograms representing an occurrence of RHOM_14625 gene fragment among studied groups. (B) Interaction plot shows the difference between the frequencies of RHOM_14625 gene fragment occurrence in studied groups. The association is seen between: i) females with IBS and males with IBS; ii) females with IBS and females without IBS; iii) females with IBS and males without IBS. 

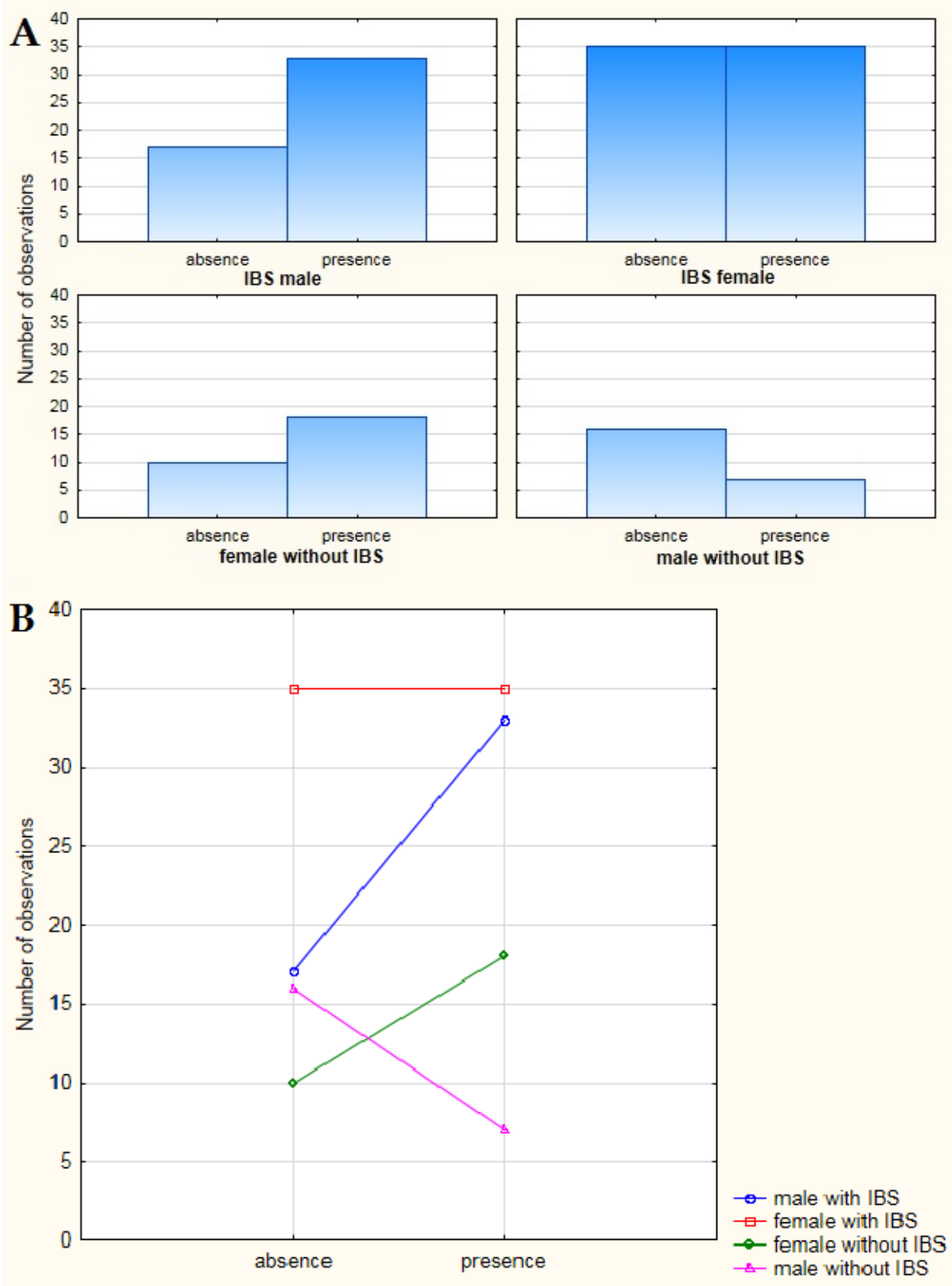

Figure 2. (A) Histograms representing an occurrence of RHOM_14635 gene fragment among studied groups. (B) Interaction plot shows the difference between the frequencies of RHOM_14635 gene fragment occurrence in studied groups. The interaction is seen between females without IBS and males with IBS.

\section{Conclusions}

Further molecular studies are necessary to evaluate the role of Roseburia hominis in intestinal microbiome in IBS patients. Taking into consideration the obtained results, it can be assumed that $R$. hominis might play a role in the assessed microbiome in IBS etiology.

Funding: This work was supported by the National Science Center in Poland, grant number: 2016/21/N/NZ5/01423 (to: Paulina Pecyna).

Conflicts of Interest: The authors declare no conflict of interest.

\section{References}

1. Thursby, E.; Juge, N. Introduction to the human gut microbiota. Biochem. J.2017, 474, 1823-1836, doi:10.1042/BCJ20160510. 
2. Kho, Z.Y.; Lal, S.K. The Human Gut Microbiome - A Potential Controller of Wellness and Disease. Front. Microbiol.2018, 9, doi:10.3389/fmicb.2018.01835.

3. Menees, S.; Chey, W. The gut microbiome and irritable bowel syndrome. F1000Research 2018, 7, doi:10.12688/f1000research.14592.1.

4. Milani, C.; Duranti, S.; Bottacini, F.; Casey, E.; Turroni, F.; Mahony, J.; Belzer, C.; Delgado Palacio, S.; Arboleya Montes, S.; Mancabelli, L.; et al. The First Microbial Colonizers of the Human Gut: Composition, Activities, and Health Implications of the Infant Gut Microbiota. Microbiol. Mol. Biol. Rev. MMBR2017, 81, doi:10.1128/MMBR.00036-17.

5. Tamanai-Shacoori, Z.; Smida, I.; Bousarghin, L.; Loreal, O.; Meuric, V.; Fong, S.B.; Bonnaure-Mallet, M.; Jolivet-Gougeon, A. Roseburia spp.: a marker of health? Future Microbiol.2017, 12, 157-170, doi:10.2217/fmb-2016-0130.

6. Valdes, A.M.; Walter, J.; Segal, E.; Spector, T.D. Role of the gut microbiota in nutrition and health. BMJ2018, 361, doi:10.1136/bmj.k2179.

7. Travis, A.J.; Kelly, D.; Flint, H.J.; Aminov, R.I. Complete Genome Sequence of the Human Gut SymbiontRoseburiahominis. Genome Announc.2015, 3, doi:10.1128/genomeA.01286-15.

8. Barcenilla, A.; Pryde, S.E.; Martin, J.C.; Duncan, S.H.; Stewart, C.S.; Henderson, C.; Flint, H.J. Phylogenetic relationships of butyrate-producing bacteria from the human gut. Appl. Environ. Microbiol.2000, 66, 16541661, doi:10.1128/aem.66.4.1654-1661.2000.

9. Aminov, R.I.; Walker, A.W.; Duncan, S.H.; Harmsen, H.J.M.; Welling, G.W.; Flint, H.J. Molecular Diversity, Cultivation, and Improved Detection by Fluorescent In Situ Hybridization of a Dominant Group of Human Gut Bacteria Related to Roseburia spp. or Eubacteriumrectale. Appl. Environ. Microbiol.2006, 72, 6371-6376, doi:10.1128/AEM.00701-06.

10. Patterson, A.M.; Mulder, I.E.; Travis, A.J.; Lan, A.; Cerf-Bensussan, N.; Gaboriau-Routhiau, V.; Garden, K.; Logan, E.; Delday, M.I.; Coutts, A.G.P.; et al. Human Gut SymbiontRoseburiahominis Promotes and Regulates Innate Immunity. Front. Immunol.2017, 8, doi:10.3389/fimmu.2017.01166.

11. Takahashi, K.; Nishida, A.; Fujimoto, T.; Fujii, M.; Shioya, M.; Imaeda, H.; Inatomi, O.; Bamba, S.; Sugimoto, M.; Andoh, A. Reduced Abundance of Butyrate-Producing Bacteria Species in the Fecal Microbial Community in Crohn's Disease. Digestion2016, 93, 59-65, doi:10.1159/000441768.

12. Chassard, C.; Dapoigny, M.; Scott, K.P.; Crouzet, L.; Del’homme, C.; Marquet, P.; Martin, J.C.; Pickering, G.; Ardid, D.; Eschalier, A.; et al. Functional dysbiosis within the gut microbiota of patients with constipated-irritable bowel syndrome. Aliment. Pharmacol. Ther.2012, 35, 828-838, doi:10.1111/j.13652036.2012.05007.x.

13. Machiels, K.; Joossens, M.; Sabino, J.; De Preter, V.; Arijs, I.; Eeckhaut, V.; Ballet, V.; Claes, K.; Van Immerseel, F.; Verbeke, K.; et al. A decrease of the butyrate-producing species Roseburiahominis and Faecalibacteriumprausnitzii defines dysbiosis in patients with ulcerative colitis. Gut2014, 63, 1275-1283, doi:10.1136/gutjnl-2013-304833.

14. Chen, L.; Wang, W.; Zhou, R.; Ng, S.C.; Li, J.; Huang, M.; Zhou, F.; Wang, X.; Shen, B.; A Kamm, M.; et al. Characteristics of fecal and mucosa-associated microbiota in Chinese patients with inflammatory bowel disease. Medicine (Baltimore)2014, 93, e51, doi:10.1097/MD.0000000000000051.

15. Rigsbee, L.; Agans, R.; Shankar, V.; Kenche, H.; Khamis, H.J.; Michail, S.; Paliy, O. Quantitative profiling of gut microbiota of children with diarrhea-predominant irritable bowel syndrome. Am. J. Gastroenterol.2012, 107, 1740-1751, doi:10.1038/ajg.2012.287. 
16. Roberfroid, M.; Gibson, G.R.; Hoyles, L.; McCartney, A.L.; Rastall, R.; Rowland, I.; Wolvers, D.; Watzl, B.; Szajewska, H.; Stahl, B.; et al. Prebiotic effects: metabolic and health benefits. Br. J. Nutr.2010, 104, S1-S63, doi:10.1017/S0007114510003363.

17. Soldi, S.; Vasileiadis, S.; Uggeri, F.; Campanale, M.; Morelli, L.; Fogli, M.V.; Calanni, F.; Grimaldi, M.; Gasbarrini, A. Modulation of the gut microbiota composition by rifaximin in non-constipated irritable bowel syndrome patients: a molecular approach. Clin. Exp. Gastroenterol.2015, 8, 309-325, doi:10.2147/CEG.S89999.

Publisher's Note: MDPI stays neutral with regard to jurisdictional claims in published maps and institutional affiliations.

(C) 2020 by the authors. Submitted for possible open access publication under the terms and conditions of the Creative Commons Attribution (CC BY) license (http://creativecommons.org/licenses/by/4.0/). 